\title{
Le Role de la Litterature dans le Developpement de la Nation
}

\author{
Theodora Onuko*
}

\section{Resume}

La littérature est une réflexion de la vie, des réalités d'un peuple donné exprimées et réalisées par les moyens du langage orale ou écrit.Elle vise souvent à une valeur esthétique et cherche à faire plaisir. C'est un produit de l'imagination et son but ultime est de faire le lecteur éprouver du plaisir en même temps qu'il le moralise. Elle fait nâ̂tre dans ses lecteurs ou dans son audience des sentiments de joie ou de douleur pendant qu'ils comprennent et assimilent les idées qui y sont exprimées.Cette communication a l'objectif d'étudier les rôles que peut jouer la littérature dans le développement et la formation d'une société telle que le Nigéria. Nous mettrons en relief les rôles significatifs joués par les sous- genres de la littérature orale et écrite pour montrer que la littérature est inséparable de la société. http://dx.doi.org/10.4314/ujah.v13i1.11

\section{Abstract}

Literature is a reflection of life, the socio-cultural realities of a given people expressed through oral or written language. It has the double purpose of instructing and pleasing. It s a work of imagination and its ultimate goal is to instruct and inculcate moral virtues in its reader. It tends to sensitize the reader to reflect and speculate on certain strange phenomenon. The objective of this paper is to examine the roles of literature in the development and progress of any society. The fundamental roles played by the oral and the written aspects are analyzed in order to prove that literature is inseparable from the society. 


\section{Introduction}

Pour Wikipedia "le terme littérature n'a pas eu la même signification qu'on lui reconnaît au moment actuel" (L'encyclopédie libre, 4 novembre. 2006). Milly montre que le sens change selon la différente conception qu'on lui fait à chaque époque et dans chaque pays" (29,La littérature peut se définir d'après le dictionnaire Petit Larousse comme $<<$ l'ensemble de œuvres orales ou écrites auxquelles on reconnaît une finalité esthétique>>(246). Elle s'intéresse à l'histoire ,à la religion, à la culture, à la mort, aux normes, aux valeurs, et à tous les donnés qui appartiennent aux réalités socio-culturelles, politiques et économiques d'une société.

La littérature est souvent exprimée par des énoncés ou bien par des mots qui sont composés de deux parties: le signifiant et le signifié ,c'est-à dire la forme et le fond. Ce sont les idées ou le sens qui sont contenus dans le signifié, mais les effets esthétiques et émotionnelles se réalisent à la manière dont les idées sont organisées dans les éléments linguistiques. Wellek et Warren expliquent que si ces événements ne sont pas bien exprimés, ils peuvent ne pas provoquer aucun effet sur le lecteur ou sur l'audience (195).Dans tout cas,il existent toutes sortes de littérature selon les langues, les continents, les pays d'origine. Il y a par exemple, la littérature anglophone, la littérature francophone, la littérature allemande, la littérature chinoise etc. Milly montre que "chaque littérature s'intéresse à la société, à ses milieux particulièrs, à ses rouages et à son évolution" (31). Culler remarque que:

Dans ces conditions, on pourrait conclure que la littérature n'est rien d'autre que ce qu'une société donnée traite comme de la littérature: c'est-à-dire un ensemble de textes que les arbitres de la culture - les professeurs, les écrivains, les critiques, les académiciens 
reconnaissent comme appartenant à la

littérature. (32).

D'après Wellek et Warren "une grande partie des questions soulevées par l'étude de la littérature sont, du moins en dernière analyse ou par implication, des questions sociales: questions de traditions et de conventions, normes et genres, symboles et mythes." (129).Alors les objets d'étude de la littérature se basent souvent sur des événements économiques, des faits sociaux et politiques, c'est-à-dire certains aspects de la réalité sociale. (Wellek et Warren 132). Puisque la nature d'un objet émane de l'usage qu'on en fait, on comprendra la nature et la fonction de littérature par son usage du langage. On peut comparer la littérature à un ornement de valeur fait du langage tandis qu'un monument est bâti de pierres précieuses comme la bronze ou le marbre.

Nous notons que la nature et la fonction de littérature se modifient et se transforment selon les époques et les cultures données. L'auteur d'une œuvre littéraire pourra chercher à présenter sa propre perception de la réalité et de sa vision du monde. Wellek et Warren remarquent également qu'un écrivain littéraire, en tant que critique sociale devrait avoir un prise de conscience des situations particulières. Il doit partager l'attitude et l'idéologie particulières du critique luimême. (130). Il tend souvent à projeter ses convictions, ses émotions, ses croyances et, même ses réactions. Son œuvre pourrait persuader le lecteur d'accepter ses vues personnelles. Arnold Gerald remarque aussi que $<<$ la littérature est un moyen de communication qui doit aider les hommes à cerner leur époque, à mieux comprendre ce monde vénéneux et violent $>>(<$ http :sitevoila.fr./001 >). Nous analyserons donc les rôles significatifs que joue la littérature dans les processus de développement social.

Robert Pour Tous, dictionnaire de la langue française définit le terme comme $<1$.Action de développer, de donner 
toute son étendue à quelque chose.2.Action de développer(une pellicule photographique)Fait de développer ;évolution de ce qui se développe $=$ croissance. Développement intellectuel, développement de l'esprit>>(319)Par cette définition nous comprenons que le développement signale qu'il du progrès dans différents domaines de la vie sociale. Toute société humaine est composée d'un groupement des hommes. Alors, l'homme est l'acteur principal de développement parce que c'est à travers ses actions que du progrès et des transformations pourront être réalisées dans tous les domaines : spirituels, physiques, économiques et d'autres.

Néanmoins, la littérature vise surtout le domaine spirituel, et même l'aspect intellectuel de l'homme parce qu'elle est une œuvre de l'imagination qui aide l'homme à transcender le monde physique. Dans cet état il pourra avoir des inspirations et de la créativité qui générent des nouvelles idées qui transforment sa vie spirituellement ou technologiquement. C'est en apprenant nos cultures et nos traditions que les enfants qui sont la future génération s'instruisent des valeurs qui sont bonnes pour notre société. Le vrai développement est celui qui parvient de chez-soi et de son environnement naturel. Au sujet de sous-développement au Tchad, Khalil Alio remarque que $<<$ Pour remédier à cette situation, on a opéré une sorte de retour en arrière, afin de retrouver le point de repère nécessaire pour rectifier le parcours, d'où le développement centré l'homme>>(253-254).

Il découle que la littérature, surtout la littérature africaine pourra se servir comme un instrument par lequel les Africains découvrent leur identité perdue. Peu de développement peut y être actualisé dans une société qui ne le concilie pas avec sa culture. Wellek et Warren nous expliquent aussi que "l'étude de la littérature orale fait partie intégrante des études littéraires et que l'on ne peut pas la dissocier de la littérature écrites (65). Il existe souvent une intégration constante entre les deux et, mêmes certains romans 
comme Les fables de La Fontaine contiennent des contes et des folklores. En Afrique la littérature traditionnelle s'était transmise oralement par les vieux, les griots, les poètes, les prêtres qui étaient gardiens de la tradition. La littérature orale est très importante dans la société traditionnelle africaine parce qu'elle fait partie de la vie du peuple africain. Alors, elle est transmise fidèlement de génération en génération selon ses fonctions dans la société (Cauvin 3). Elle s'intéresse à expliquer l'origine de l'homme dans la civilisation africaine, de régler la moralité dans la vie sociale et d'analyser le sens de l'existence en s'appuyant sur les traditions des ancêtres (9). D'après Cauvin:

Les contes sont dits la nuit. Après le repas du soir, les gens se rassemblent autour d'un feu, dans un cour, ou sur la place du village. Souvent les plus jeunes commencent par lancer des devinettes et chacun cherche les réponses. Puis, à un certain moment, le premier conte apparaît et la soirée peut continuer fort tard dans la nuit ... (9)

En leur racontant divers récits et histoires ils apprennent aux enfants les mœurs de la société. Certains de ces récits et histoires qui se basent sur des vertus et des leçons de l'obéissance, de la bonté, de la patience, de l'honnêteté, de l'hospitalité, aident les parents à régler les conduits de leurs enfants (Cauvin 9).De cette façon les vieux transmettent les croyances, et les expériences de leur race. Les jeunes apprennent facilement leur langage à travers l'emploi des proverbes et des métaphores par les images.

11 en va que cette type littérature est un phénomène qui se produit dans un milieu donné pour amener des changements positifs chez ceux qui y habitent. On aperçoit du développement dans une société quand les mauvaises 
circonstances qui y existaient sont transformées.

Nous croyons que le développement prend plusieurs dimensions; physique, mental et spirituel. L'homme se ressent souvent le besoin de réaliser ses ambitions dans tous ces domaines. Mais l'on ne pourra pas arriver à cette transformation si l'on ne considère pas tous les aspects de l'homme. Nous ne croyons pas que la tâche du développement appartienne exclusivement au gouvernement mais à tout le monde qui vivent dans une société donnée.C'est pourquoi il est nécessaire de sensibiliser et mobiliser tout le monde à trouver des solutions aux problèmes socio-politiques dans la société.

On devrait se rendre compte également que les œuvres littéraires des écrivains de Négritude comme Léon Damas, Senghor, Aimé Césaire, ont facilité la lutte contre la colonisation. La diffusion de leurs idées révolutionnaires a évoqué une prise de conscience chez les peuples noirs. Ensemble, ils ont revendiqué et gagné la liberté et l'indépendance pour leurs pays. Alors la littérature est un instrument par excellence pour la diffusion des faits socioculturels parmi les peuples du monde. La transmission de ces idées littéraires et culturelles s'avère efficace pour la transformation et la civilisation des mœurs de la société.

Nous croyons qu'un écrivain littéraire, en tant que critique sociale devrait avoir une prise de conscience des situations particulières. Il devrait partager des attitudes et des idéologies particulières du critique lui-même puisqu'il s'inspire souvent des problèmes et des faits socio- culturels qui se trouvent dans la société. Et nous sommes d'avis que l'homme devrait réagir contre les vices sociales pour faire des impacts dans son milieu.

Les œuvres littéraires écrites en grecque et en latin avaient beaucoup aidé à l'avancement des langues européennes et aux renseignements socio-culturels pendant la Renaissance. Beaucoup de monde avaient également adopté les vertus et les 
mœurs qui y sont exprimés pour transformer leur vie spirituelle. Inspirés par les œuvres anciennes, certains auteurs les prenaient pour modèles avec lesquels ils ont pu établir et canoniser leurs propres littératures nationales. (Salomon, 32). Evidemment, les instructions qui y sont puisées avaient aussi aidé dans la formation et l'enrichissement des idées scientifiques des humanistes à cette époque. (Bassnett Mcguire, 44).

L'écrivain littéraire, cherche souvent à critiquer les méfaits et les vices de sa société. Par leur écriture des écrivains nous révèlent certains faits historiques de leur vie personnelle et de leur société. Ils semblent d'avoir la conscience des situations sociales de leur milieu. (Wellek et Warren 130). Par exemple, Montesquieu dans Lettres Persanes dépeint la société de son temps pour critiquer certaines institutions sociales en France comme la cour, le gouvernement et l'Eglise, les foules parisiens et leur mode de vie. (Salomon 89).

La Seconde Guerre Mondiale et l'occupation ont également provoqué une littérature de résistance en France, chez les Existentialistes dont le chef est J.P. Sartre. Par leur écriture, ils ont cherché à encourager et à libérer leur peuple. (Salomon 194 - 195). Quant à la littérature, Sartre observe que «I' écrivain doit s'engager tout entier dans ses ouvrages, et non pas comme une passivité abjecte... mais comme cette totale entreprise de vivre » 40.) Chez lui écrire c'est I' engagement et la contestation contre toute oppression qui supprime la liberté de l'homme. Iwuchukwu montre aussi que:

Tout écrivain vit dans un milieu socio-culturel familier ou étranger, qui lui sert normalement de source d'inspiration productrice. Il se montre attentif à ce qu'il voit, lit et entend, notamment les discours et les pratiques en société historique qu'il s'emploierait à textualiser. (302). 
Les écrivains littéraires parfois agissent comme des visionnaires et des prophètes qui, par leur écriture mettent leur société en garde contre un malaise, un vice qui pourrait détruire toute une race ou une société. Comme des citoyens, ils ont leurs propres alliances et idéologies. Ils peuvent prendre positions sur des problèmes d'intérêts: religieux ou politiques. Certains d'entre eux peuvent prendre parti dans les grands débats politiques de leurs peuples afin de réveiller une prise de conscience dans leurs sociétés. (138).

Cela explique pourquoi, des écrivains comme Wole Soyinka et Chinua Achebe ont pris leur position dans la lutte contre la corruption au Nigéria. Plusieurs fois ils ont critiqué à travers la média les méfaits des gens au gouvernement contre leurs peuples. On comprendrait alors Chinua Achebe, lorsqu'en 2005, il a refusé d'accepter un prix d'honneur donné par le président nigérian, aux hommes qui se sont distingués dans différents domaines au Nigéria.

En réalité, la littérature pourra aider l'homme de reflêchir plus profondément sur sa vie et à celles d'autres dans sa société .C'est pourquoi l'on ne s'étonnerait pas trop de voir qu'Achebe a refusé cet honneur comme un proteste contre la marginalisation de l'Etat d'Anambra par le gouvernement nigérian, car lui même et d'origine de cet état en question. Et Wole Soyinka a, à plusieurs moments, critiqué les manœuvres de Chef Obasanjo de devenir, pour la troisième fois le président du Nigéria.Ces attitudes et ces réactions d'Achebe et de Soyinka sont révelées dans leurs idées de l'écrivain africain vis-à-vis de sa société. D'après Ogungbesan, Achebe a soutenu ce fait en remarquant que "the writer's duty is to explore in depth the human condition. In Africa he cannot perform this task unless he has a proper sense of history." (2).

$\mathrm{Au}$ moment actuel, les écrivains de l'Afrique occidentale écrivent des œuvres littéraires pour critiquer les questions du racisme, du conflit de générations, de la 
corruption, de la misère, de la guerre et de l'émancipation des femmes parce qu'une fois que l'indépendance est acquise, les dirigéants s'occupent à chercher seulement leur intérêt politique.Nubukpo Komla soutient ce rôle de littérature comme le véhicule de l'engagement par le biais de cette citation $:<<$ La littérature créatrice dans une modeste mesure participe de ce combat, et la critique littérature qui la suit partout comme son ombre et l'oriente souvent, clarifie et renouvelle sans cesse la force et le sens de son impact sur les lecteurs potentiels ou réels $>>(127)$. Les écrivains littéraires de l'origine africaine visent vraiment à effectuer des changements socio-culturels par leurs œuvres critiques.

ll va sans dire que l'on ne peut pas aliéner la littérature de la vie sociale parce que c'est une institution sociale qui exprime la vie d'un peuple et d'une époque donnée. Elle a des fonctions sociales. Elle montre et dépeint des événements et des réalités sociales exprimées par le langage qui est en lui même un instrument social. Les idées et les philosophies qui y sont représentées sont des faits historiques ou des vraisemblances de la réalité du passé, et du présent. (Wellek et Warren, 129).Elle est active pour apporter des transformations dans plusieurs circonstances de la société.

\section{Conclusion}

Dans cette communication nous avons établi que la littérature est une réflexion de la vie, des réalités d'un peuple donné exprimées et réalisées par les moyens du langage oral ou écrit. Elle vise souvent à une valeur esthétique et cherche à faire plaisir. On aperçoit du développement dans une société quand les mauvaises circonstances qui y existaient sont transformées.

Cependant, nous croyons que le développement prend plusieurs dimensions ; physique, mental et spirituel. L'homme se ressent souvent le besoin de réaliser ses ambitions dans tous ces domaines. Mais l'on ne pourra pas arriver à cette 
transformation si l'on ne considère pas tous les aspects de l'homme. Nous ne croyons pas que la tâche du développement appartienne exclusivement au gouvernement mais à tout le monde dans la société.C'est pourquoi il est nécessaire de sensibiliser et mobiliser tout le monde à trouver des solutions aux problèmes socio-politiques dans la société. Et la littérature s'avère efficace pour cette tâche.

$\mathrm{Au}$ cours des âges, la littérature a beaucoup contribué au processus de la civilisation. Alors la littérature est un instrument par excellence pour la diffusion des faits socioculturels parmi les peuples du monde. La transmission de ces idées littéraires et culturelles s'avère efficace pour la transformation et la civilisation des mœurs de la société. L'écrivain littéraire devrait partager l'attitude et l'idéologie particulières du critique lui-même puisqu'il s'inspire souvent des problèmes et des faits socio- culturels qui se trouvent dans sa société. Et nous sommes du cet avis parce que l'on devrait réagir contre les vices sociales pour faire des impacts dans son milieu.

* Dr. Theodora Onuko lectures in the Department of Modern European Languages, Nnamdi Azikiwe University, Awka. 


\section{Quvres Citees}

Alio Khalil, "Langues, Démocratie et Développement: Préalables à un aménagement linguistique au Tchad" dans Ajulo, S et The Fetschrift Committee eds, Language and Society, Lagos, University Press, 2000.

Bassnett-Mcguire, Susan Translation Studies, London and Newyork, Routledge, 1992.

Cauvin, Jean, Comprendre Les Contes, Versailles, Les Classiques Africains, 1992.

Culler, Jonathan, "La Littérarité" La Théorie Littéraire:

Problèmes et Perspectives, Angenot, Marc et al, eds. Paris, Presses Universitaires de France 1989.

Komla, Nubukpo, $<<$ Mondialisation et critique littéraire >>dans Particip'Action : Revue interafricaine de littérature et philosophie, les presses de l'imprimerie Saint- Louis,Togo,Lomé,2009.

Iwuchukwu, Matthew. "Théorie Littéraire et Sociocritique: Pour une Médiation Théorique et Methodilogique dans Nsukka Journal of the Humanities, Faculty of Arts, University of Nigeria, 2002.

Milly, Jean. Fac Littérature: Poétique des Textes, Paris, Editions Nathan, 1992.

Ogungbesan, Kolawole. New West African Literature, London, Heinemann Educational Books Ltd, 1979.

Salomon, Pierre. Littérature Française, Paris, Bordas, 1993.

Sartre, Jean-Paul. Qu'est-ce que la littérature? Paris, Editions Gallimard, 1948.

Wellek, René et Warren, Austin. La Théorie Littéraire. Traduit de l'Anglais par Audigier. Jean-Pierre et Cattegno, Jean, Paris, Editions du Seuil, 1971.

Robert Pour Tous: Dictionnaire de la langue française, Paris, 
Dictionnaire le Robert, 1994.

Littérature Wikipedia. L'encyclopédie libre 4 novembre. 2006). <http://en. Wikipedia.org/Wiki>>>

(<http. Arnold Gerald:sitevoila.fr./001>). 